\title{
Immature-like molecular expression patterns in the hippocampus of a mouse model of dementia with Lewy body-linked mutant $\beta$-synuclein
}

\author{
Hideo Hagihara ${ }^{1}$ (D), Masayo Fujita ${ }^{2}$ (D) Juzoh Umemori ${ }^{1}$ (D), Makoto Hashimoto ${ }^{3}$ (D) and Tsuyoshi Miyakawa $^{1 *}$ (D)
}

\begin{abstract}
Aim: Maturation abnormalities of the brain cells have been suggested in several neuropsychiatric disorders, including schizophrenia, bipolar disorder, autism spectrum disorders, and epilepsy. In this study, we examined the expression patterns of neuronal maturation markers in the brain of a mouse model of dementia with Lewy bodylinked mutant $\beta$-synuclein ( $\beta S$ ), especially in the hippocampus, to explore whether such brain abnormalities occur in neurodegenerative disorders as well.

Methods: Quantitative PCR ( $\mathrm{qPCR}$ ) and immunohistochemical analyses were performed using the hippocampus of 14-month-old P123H BS transgenic (Tg) mice to evaluate the expression of molecular markers for maturation of dentate granule cells.

Results: Based on QPCR results, expression of Tdo2 and Dsp (markers of mature granule cells) was decreased and that of Drd1a (a marker of immature granule cells) was increased in the hippocampus of P123H $\beta S \mathrm{Tg}$ mice compared to that in wild-type controls. Immunohistochemical analysis revealed decreased expression of mature granule cell markers Calb1 and Gria1, along with increased expression of the microglial marker Ibal, in the hippocampal dentate gyrus region of $\mathrm{P} 123 \mathrm{H}$ BS Tg mice. P123H BS Tg mice exhibited immature-like neuronal molecular expression patterns and microgliosis in the hippocampus. Pseudo-immaturity of dentate granule cells, associated with neuroinflammation, may be a shared endophenotype in the brains of at least a subgroup of patients with neuropsychiatric disorders and neurodegenerative diseases.
\end{abstract}

Keywords: $\beta$-Synuclein, Endophenotype, Hippocampus, Immature dentate gyrus, Neurodegenerative disorders

\section{Main text}

"Immature dentate gyrus (iDG)" phenotype is commonly found in several mouse models of neuropsychiatric disorders [1], including schizophrenia/intellectual disability [2], bipolar disorder [3], and epilepsy [4, 5]. In this phenotype, almost all granule cells in the adult hippocampal DG are arrested in a pseudo-immature state, in terms of molecular and electrophysiological characteristics. More specifically, molecular features of iDG include a decrease in expression of mature granule cell markers (e.g.,

\footnotetext{
* Correspondence: miyakawa@fujita-hu.ac.jp

${ }^{1}$ Division of Systems Medical Science, Institute for Comprehensive Medical

Science, Fujita Health University, 1-98 Dengakugakubo Kutsukake-cho,

Toyoake, Aichi 470-1192, Japan

Full list of author information is available at the end of the article
}

tryptophan 2,3-dioxygenase [Tdo2], Desmoplakin [Dsp], and Calbindin 1 [Calb1] [6]) along with an increase in the expression of immature granule cell marker (dopamine receptor D1 [Drd1a]) [1]. iDG-like phenotype was also observed in patients with schizophrenia and bipolar disorder [7]. As for neurodegenerative disorders, recent studies revealed that Alzheimer's disease mouse model showed a drastic decrease in the expression of Calb1 in adult DG, suggestive of iDG-like phenotype in the mouse model $[8,9]$. In the present study, we investigated whether a mouse model of dementia with Lewy body-linked mutant $\beta$-synuclein (P123H $\beta$ S) exhibits iDG-related molecular features. The $\mathrm{P} 123 \mathrm{H} \beta \mathrm{S}$ transgenic $(\mathrm{Tg})$ mouse showed many behavioral abnormalities, including hyperlocomotor

(C) The Author(s). 2018 Open Access This article is distributed under the terms of the Creative Commons Attribution 4.0 International License (http://creativecommons.org/licenses/by/4.0/), which permits unrestricted use, distribution, and 
activity, impairment of nest building, and impaired spatial memory, in the middle stage (6-10 months of age), before the onset of motor dysfunction that became apparent in the later stage (12-18 months) [10,11]. In the brain of these mice, neuritic pathologies such as $\beta S$ accumulation and axonal swellings, and astrogliosis were observed in various regions, including hippocampus, during the middle to late stage [10]. However, maturation abnormalities in the brain have not been examined in these mice.

Whole hippocampus of the mouse (14 months of age) was dissected out and quantitative PCR (qPCR) analysis was conducted to examine mRNA expression levels of Drd1a, Bdnf, Tdo2, Dsp, and Calb1. The detailed method for qPCR is described in the Additional file 1. Expression of Drd1a was significantly increased and that of $B d n f$ showed an increasing trend in the hippocampus of $\mathrm{P} 123 \mathrm{H}$ BS $\mathrm{Tg}$ mice compared to that in wild-type mice (Fig. 1a, Additional file 1: Table S1). Expression of
Tdo2 and Dsp was significantly decreased in the $\mathrm{P} 123 \mathrm{H}$ $\beta S \mathrm{Tg}$ mice while that of Calb1 was comparable between $\mathrm{P} 123 \mathrm{H} \beta \mathrm{S} \mathrm{Tg}$ and wild-type mice. To assess Calb1 expression, focusing on the DG region, we conducted immunohistochemical analyses (see Additional file for the detailed method). The results showed a significant decrease in Calb1 expression in the DG granule cell layer of $\mathrm{P} 123 \mathrm{H} \beta S \mathrm{Tg}$ mice (Fig. 1b, Additional file 1: Figure S1). Notably, a patch-like reduction of Calb1 immunoreactivity was observed in the DG granule cell layer of $\mathrm{P} 123 \mathrm{H} \beta \mathrm{S} \mathrm{Tg}$ mice, which may not have been due to apparent cell loss, since nuclear staining was observed uniformly throughout the DG granule cell layer. We also found a decrease in immunoreactivity for Gria1, whose expression increased with maturation of granule cells [12], but decreased in the DG of typical mouse models with iDG [12], and in that of P123H $\beta S$ Tg mice (Fig. 1c). In addition, immunoreactivity for ionized calcium binding adaptor molecule 1 (Iba1) was increased in
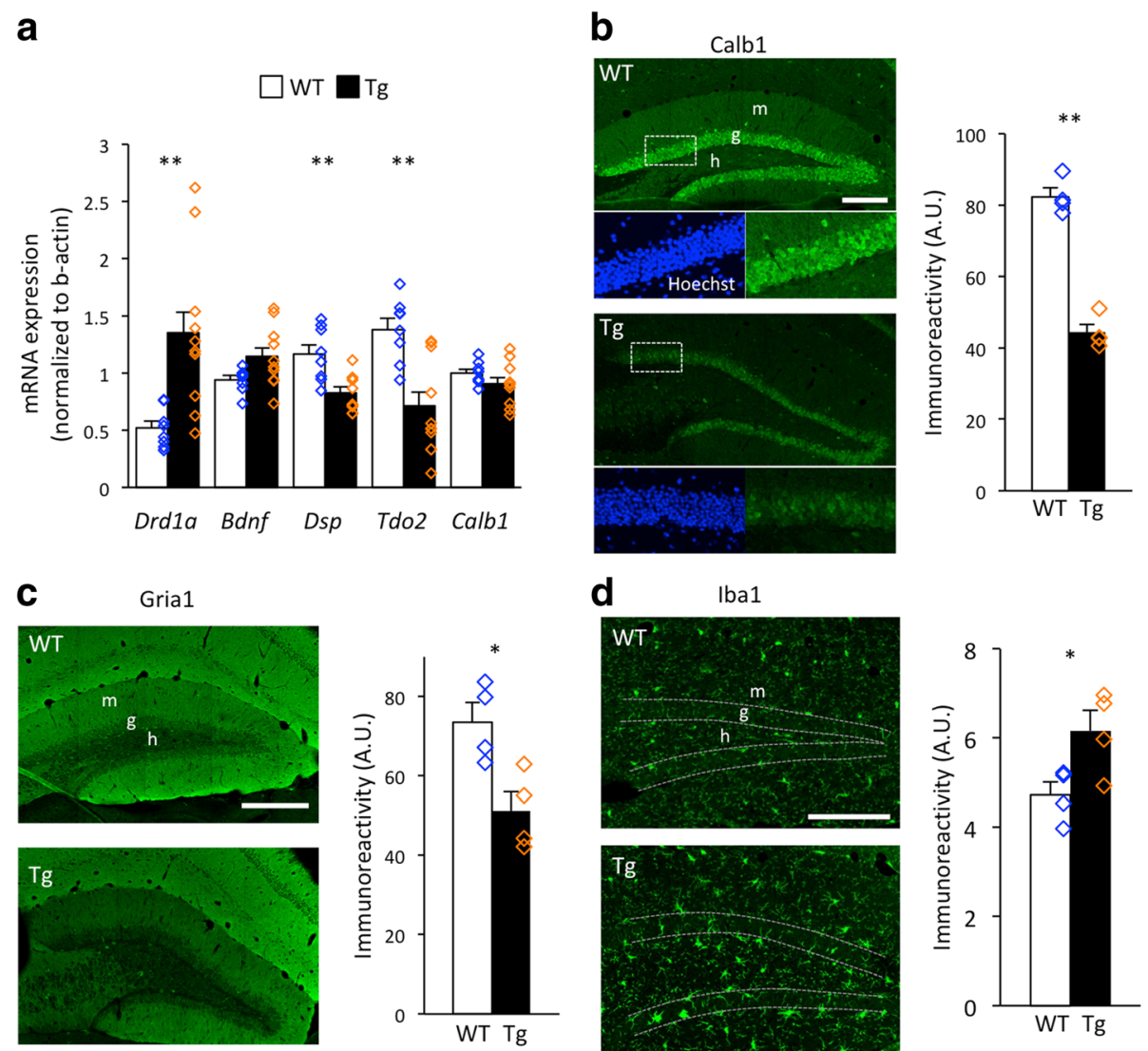

Fig. 1 iDG-like molecular phenotypes in the hippocampus of P123H BS Tg mice. a Results of quantitative PCR. Bar graphs represent relative mRNA expression levels normalized to $\beta$-actin mRNA. Data obtained from two independent experiments were combined and shown as the mean \pm SEM. ( $n=8$ for wild-type mice and $n=10$ for P123H BS Tg mice). ${ }^{*} P<0.05,{ }^{*} P<0.01$ versus wild-type mice. b-d Representative images (left panels) and the semi-quantitative results (right panels) of immunofluorescence imaging of Calb1 (b), Gria1 (c), and Iba1 (d) in the DG of wildtype and P123H BS Tg mice. Higher magnification of the boxed area is shown below the corresponding panel (b). Data are shown as the mean $\pm \operatorname{SEM}\left(n=4\right.$ for each genotype). ${ }^{*} P<0.05,{ }^{* *} P<0.01$ versus wild-type mice. Scale bars, $200 \mu \mathrm{m}(\mathbf{b}, \mathbf{d})$ and $500 \mu \mathrm{m}(\mathbf{c})$. g, granule cell layer; $\mathrm{h}$, hilus; $m$, molecular layer 
the DG of P123H $\beta S$ Tg mice (Fig. 1d), suggesting that microglia are activated in the DG of these mice.

We found a significant decrease in Calb1 expression in the DG granule cell layer by immunohistochemical analysis. However, the extent of this reduction was low relative to that found in other mouse models with iDG, such as Camk2a $a^{+/-}$mice, Shn2 KO mice, and mutant Snap25 knock-in mice, whose Calb1 expression in the DG granule cell layer was almost completely depleted [2-4]. Therefore, the present qPCR analysis of whole hippocampus samples might have failed to detect a decrease in Calb1 expression in P123H $\beta S$ Tg mice, due to the presence of cells (other than granule cells) that express Calb1, such as pyramidal cells in the Ammon's horn region and particular types of interneurons that exist throughout the brain. The discrepancy between mRNA and protein levels of Calb1 may also be accounted for by some post-transcriptional mechanisms and/or differences in their half lives [13].

Patch-like reduction of Calb1 expression in the DG granule cell layer was found in $\mathrm{P} 123 \mathrm{H} \beta \mathrm{S}$ Tg mice; a similar phenotype was observed in a mouse model of Alzheimer's disease (line J20) $[8,9]$. In those papers, it was suggested that Calb1 downregulation was induced by seizure activity in patients and mouse models $[8,9]$. Patch-like reduction of Calb1 in the DG has been observed in epilepsy models [5]. Considering that epileptic seizures have been observed in $\mathrm{P} 123 \mathrm{H}$ BS $\mathrm{Tg}$ mice (unpublished observation), seizure activity might have caused Calb1 downregulation in these $\mathrm{Tg}$ mice. Interestingly, patch-like reduction of Calb1 in the DG have also been found in adult mice treated with antidepressant fluoxetine [14] and electroconvulsive stimulation [15]. Assuming that typical models showing robust

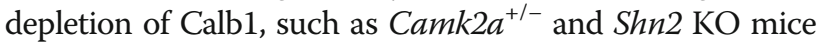
$[2,3]$, display iDG phenotypes developmentally generated, there is the possibility that these weaker phenotypes are features of iDG that are induced by dematuration in adults.

In conclusion, $\mathrm{P} 123 \mathrm{H} \beta \mathrm{S}$ Tg mice exhibited iDG-like signatures and microgliosis in the DG. It would be of interest to determine the time of appearance of the iDG phenotype in these mice in relation to that of behavioral abnormalities.

\section{Additional file}

Additional file 1: Materials and Methods. Table S1. Raw data of $\mathrm{qPCR}$ analysis (average $\mathrm{Cq}$ value). Figure $\mathbf{S 1}$. Immunohistochemical images used for quantitative analysis in this study. (DOCX $25253 \mathrm{~kb}$ )

\footnotetext{
Abbreviations

Bdnf: Brain-derived neurotrophic factor; Calb1: Calbindin; Camk2a: Calcium/ calmodulin-dependent protein kinase II alpha; DG: Dentate gyrus; Drd1a: Dopamine receptor D1; Dsp: Desmoplakin; Gria1: Glutamate receptor, ionotropic, AMPA 1; Iba1: Ionized calcium binding adaptor molecule 1; iDG: Immature dentate gyrus; PBS: Phosphate-buffered saline: PCR: Polymerase chain reaction; Shn2: Schnurri-2; Snap25: Synaptosomalassociated protein, 25 kDa; Tdo2: Tryptophan 2,3-dioxygenase; $\beta$ S: $\beta$ synuclein
}

Acknowledgements

We thank the members of Hashimoto lab for the animal husbandry.

Funding

This work was supported by the Grants-in-Aid for Scientific Research (JP25242078) from Japan Society for the Promotion of Science; and the Grant-in-Aid for Scientific Research on Innovative Areas (JP24111546, JP24111555, JP25116526, and JP16H06462) from the Ministry of Education, Culture, Sports, Science and Technology.

Availability of data and materials

All data are available in the Additional file 1.

\section{Authors' contributions}

$\mathrm{HH}, \mathrm{MF}, \mathrm{MH}$, and TM conceived and designed the experiments. $\mathrm{HH}$ and $\mathrm{TM}$ wrote the article. MF prepared the samples; $\mathrm{HH}$ and JU performed molecular experiments and analyzed the data. All authors read and approved the final manuscript.

\section{Ethics approval}

The experimental procedures and housing conditions were approved by the Institutional Animal Care and Use Committee at Tokyo Metropolitan Institute of Medical Science (Setagaya-ku, Tokyo, Japan). Frozen or fixed mouse brain samples were prepared at Tokyo Metropolitan Institute of Medical Science and transported to Fujita Health University (Toyoake, Aichi, Japan) for analysis.

Consent for publication

Not applicable

\section{Competing interests}

The authors declare that they have no competing interests.

\section{Author details}

'Division of Systems Medical Science, Institute for Comprehensive Medical Science, Fujita Health University, 1-98 Dengakugakubo Kutsukake-cho, Toyoake, Aichi 470-1192, Japan. ${ }^{2}$ Addictive Substance Project, Tokyo Metropolitan Institute of Medical Science, Setagaya-ku, Tokyo 156-8506, Japan. ${ }^{3}$ Laboratory of Parkinson's Disease, Tokyo Metropolitan Institute of Medical Science, Setagaya-ku, Tokyo 156-8506, Japan.

Received: 1 May 2018 Accepted: 15 June 2018

Published online: 06 July 2018

\section{References}

1. Hagihara H, Takao K, Walton NM, Matsumoto M, Miyakawa T. Immature dentate gyrus: an endophenotype of neuropsychiatric disorders. Neural Plasticity. 2013;2013:318596.

2. Takao K, Kobayashi K, Hagihara H, Ohira K, Shoji H, Hattori S, et al. Deficiency of schnurri-2, an MHC enhancer binding protein, induces mild chronic inflammation in the brain and confers molecular, neuronal, and behavioral phenotypes related to schizophrenia. Neuropsychopharmacology. 2013;38:1409-25.

3. Yamasaki N, Maekawa M, Kobayashi K, Kajii Y, Maeda J, Soma M, et al. Alpha-CaMKII deficiency causes immature dentate gyrus, a novel candidate endophenotype of psychiatric disorders. Molecular Brain. 2008;1:6.

4. Ohira K, Kobayashi K, Toyama K, Nakamura HK, Shoji H, Takao K, et al. Synaptosomal-associated protein 25 mutation induces immaturity of the dentate granule cells of adult mice. Molecular Brain. 2013;6:12.

5. Shin R, Kobayashi K, Hagihara H, Kogan JH, Miyake S, Tajinda K, et al. The immature dentate gyrus represents a shared phenotype of mouse models of epilepsy and psychiatric disease. Bipolar Disorders. 2013;15:405-21.

6. Radic T, Frieß L, Vijikumar A, Jungenitz T, Deller T, Schwarzacher SW. Differential postnatal expression of neuronal maturation markers in the dentate gyrus of mice and rats. Front Neuroanat. 2017;11:104.

7. Walton NM, Zhou Y, Kogan JH, Shin R, Webster M, Gross AK, et al. Detection of an immature dentate gyrus feature in human schizophrenia/bipolar patients. Translational Psychiatry. 2012;2:e135.

8. Palop JJ, Chin J, Roberson ED, Wang J, Thwin MT, Bien-Ly N, et al. Aberrant excitatory neuronal activity and compensatory remodeling of inhibitory hippocampal circuits in mouse models of Alzheimer's disease. Neuron. 2007; 55:697-711. 
9. You JC, Muralidharan K, Park JW, Petrof I, Pyfer MS, Corbett BF, et al. Epigenetic suppression of hippocampal calbindin-D28k by $\Delta$ FosB drives seizure-related cognitive deficits. Nature Medicine. 2017;23:1377-83.

10. Fujita M, Sugama S, Sekiyama K, Sekigawa A, Tsukui T, Nakai M, et al. A Bsynuclein mutation linked to dementia produces neurodegeneration when expressed in mouse brain. Nature Communications. 2010;1:110.

11. Fujita M, Hagino Y, Takamatsu Y, Shimizu Y, Takamatsu Y, Ikeda K, Hashimoto H. Early manifestation of depressive-like behavior in transgenic mice that express dementia with Lewy body-linked mutant $\beta$-synuclein. Neuropsychopharmacology Reports. 2018;38:95-97.

12. Hagihara H, Ohira K, Toyama K, Miyakawa T. Expression of the AMPA receptor subunits GluR1 and GluR2 is associated with granule cell maturation in the dentate gyrus. Front Neurosci. 2011;5:100.

13. Greenbaum D, Colangelo C, Williams K, Gerstein M. Comparing protein abundance and mRNA expression levels on a genomic scale. Genome Biology. 2003:4:117.

14. Kobayashi K, Ikeda Y, Sakai A, Yamasaki N, Haneda E, Miyakawa T, Suzuki H. Reversal of hippocampal neuronal maturation by serotonergic antidepressants. PNAS. 2010;107:8434-9.

15. Imoto Y, Segi-Nishida E, Suzuki H, Kobayashi K. Rapid and stable changes in maturation-related phenotypes of the adult hippocampal neurons by electroconvulsive treatment. Molecular Brain. 2017;10:8.

Ready to submit your research? Choose BMC and benefit from:

- fast, convenient online submission

- thorough peer review by experienced researchers in your field

- rapid publication on acceptance

- support for research data, including large and complex data types

- gold Open Access which fosters wider collaboration and increased citations

- maximum visibility for your research: over $100 \mathrm{M}$ website views per year

At BMC, research is always in progress.

Learn more biomedcentral.com/submissions 